\title{
Características sociodemográficas e clínicas de uma coorte de puérperas em um
}

\section{hospital de Santa Catarina}

\author{
Sociodemographic and clinical characteristics of a cohort of puerperal women in a hospital in Santa \\ Catarina
}

Características sociodemográficas y clínicas de una cohorte de puérperas en un hospital de Santa Catarina

Recebido: 17/12/2020 | Revisado: 26/12/2020 | Aceito: 14/01/2021 | Publicado: 17/01/2021

\author{
Felipe Ongaratto \\ ORCID: https://orcid.org/0000-0002-9763-9104 \\ Universidade Federal da Fronteira Sul, Brasil \\ E-mail: felipeongarato@gmail.com \\ Sofia Belfort Bomfim \\ ORCID: https://orcid.org/0000-0002-5701-7298 \\ Universidade Federal da Fronteira Sul, Brasil \\ E-mail: sofia.belfort.b@gmail.com \\ Maíra Rossetto \\ ORCID: https://orcid.org/0000-0002-5683-4835 \\ Universidade Federal da Fronteira Sul, Brasil \\ E-mail: maira.rossetto@uffs.edu.br
}

\begin{abstract}
Resumo
O estudo objetiva detalhar as características sociodemográficas e clínicas de acordo com o tipo de parto (vaginal ou cesárea) e o tipo de convênio (SUS ou privado) de uma coorte de puérperas em um hospital público da região Oeste de SC. Trata-se de um estudo descritivo, transversal e retrospectivo, realizado a partir de dados de base de uma coorte de puérperas com partos vaginais ou cesáreas atendidas em um hospital da região oeste de Santa Catarina. A coleta dos dados ocorreu entre o período de novembro de 2019 e março de 2020 e foi feita a partir do registro dos partos vaginais e cesáreas realizados na instituição entre os meses de maio e julho de 2019. As variáveis analisadas nos prontuários foram: raça/cor/etnia e idade materna, estado civil, tipo de convênio (SUS ou privado), número de gestações, via do parto, episiotomia, laceração (primeiro ou segundo grau), comorbidades maternas, intercorrência no parto e antibioticoprofilaxia. Inferiu-se que o grupo de mulheres com faixa etária entre 26 e 30 anos foi predominante, além disso, quanto ao número de gestações, sobressaiu-se o grupo que estava na segunda gestação. A maioria das mulheres era branca, casada ou união estável, não possuía abortamento prévio, apresentou comorbidade na gestação, não teve intercorrência intraparto, utilizou o Sistema Único de Saúde, não foi submetida a episiotomia e apresentou laceração em decorrência do parto. Constatou-se uso excessivo de cesáreas, principalmente no setor privado; entretanto, o índice de realização de episiotomia converge com o que recomenda a Organização Mundial da Saúde.
\end{abstract}

Palavras-chave: Saúde da mulher; Parto; Perfil de saúde.

\begin{abstract}
The aim of this study is to describe the socio-demographics characteristics and clinical features according to the different types of birth (vaginal and caesarean section) and health care system (SUS or private) in a cohort of puerperal women from a public hospital located in the west of Santa Catarina state. This is a retrospective, descriptive and transversal study, elaborated from the data of a cohort of women who gave birth by vaginal or caesarean delivery and who were admitted in a referral hospital in the west of Santa Catarina State. The data from medical records, stored in the hospital own network, was collected between November 2019 and March 2020. The variables analysed were: race/skin colour/ethnicity, age, marital status, health system (SUS or private), number of pregnancies, delivery route, use of episiotomy, laceration (categorized in second or third degree), maternal comorbidities, complications of delivery and prophylactic antibiotic therapy. The results showed that the majority of women were between 26 and 30 years of age, were white, married or in a stable relationship, pregnant for the second or third time, never had a miscarriage, developed complications during the current pregnancy, did not have intrapartum intercurrences, utilized public health system to grant access to medical care, did not undergo episiotomy and did not have any type of perineal tears during childbirth. These results show an abusive use of caesarean section, mainly in private care system; nevertheless, the rate of episiotomy is in accordance with the Wold Health Organization recommendation.
\end{abstract}

Keywords: Women's health; Parturition; Health profile. 


\begin{abstract}
Resumen
El estudio pretende detallar las características sociodemográficas y clínicas de acuerdo con el tipo de parto (vaginal o cesárea) y el tipo de convenio (SUS o privado) de una cohorte de puérperas en un hospital público de la región Oeste de SC. Se trata de un estudio descriptivo, transversal y retrospectivo, realizado a partir de datos de base de una cohorte de puérperas con partos vaginales o cesáreas atendidas en un Hospital de la región Oeste de Santa Catarina. La recopilación de datos se llevó a cabo entre noviembre de 2019 y marzo de 2020 y se realizó a partir del registro de partos vaginales y cesáreas realizados en la institución entre los meses de mayo y julio de 2019. Las variables analizadas en los prontuarios fueron: raza/color/etnia y edad materna, estado civil, tipo de convenio (SUS o privado), número de gestaciones, vía del parto, episiotomía, laceración (primer o segundo grado), comorbidades maternas, intercorrencia en el parto y antibioticoprofilaxia. Se dedujo que el grupo de mujeres de entre 26 y 30 años fue predominante, además, en cuanto al número de gestaciones, se destacó el grupo que estaba en la segunda gestación. La mayoría de las mujeres era blanca, casada o unión estable, no poseía aborto previo, presentó comorbidad en la gestación, no tuvo intercorrencia intraparto, utilizó el Sistema Único de Salud, no fue sometida a episiotomía y presentó laceración como consecuencia del parto. Se constató uso excesivo de cesáreas, principalmente en el sector privado; sin embargo, el índice de realización de episiotomía converge con lo que recomienda la Organización Mundial de la Salud.
\end{abstract}

Palabras clave: Salud de la mujer; Parto; Perfil de salud.

\title{
1. Introdução
}

As experiências da gestação e do parto podem ser vivenciadas de diversas maneiras e terem diferentes significados para as mulheres. Embora seja esperado que o período gestacional traga as sensações de realização e de felicidade às gestantes, ele também é acompanhado de diversas alterações fisiológicas, dúvidas e expectativas, especialmente referentes ao seu desfecho: o parto (Carneiro et al., 2015). Com os avanços tecnológicos em saúde, a assistência obstétrica passou por mudanças e o parto deixou de ter a gestante como centro, passando a ser, por muitas vezes, considerado como um processo patológico em que se acredita que existe a necessidade de uma abordagem mais intervencionista (Leal et al., 2019).

Do ponto de vista clínico, os avanços na obstetrícia e o uso de tecnologias assistenciais, quando bem aplicados, são capazes de reduzir significativamente a morbimortalidade materna e fetal. No entanto, segundo a Comissão Nacional de Incorporações de Tecnologias no SUS (CONITEC), como consequência da visão patológica do parto, as mulheres e seus recém-nascidos são expostos a intervenções, por vezes, desnecessárias; dentre elas está o uso da episiotomia de rotina, uso de ocitocina e a cesárea, entre outras práticas (Ministério da Saúde [MS], 2017). Estudos afirmam que o uso indiscriminado da episiotomia no parto vaginal e a cesárea eletiva figuram como importantes fatores de risco para prematuridade, morte materna pós-parto e podem trazer consequências irreversíveis que afetam a qualidade de vida das mulheres (Esteves-Pereira et al., 2016; Jiang, Qian, Carroli, \& Garner, 2017).

Nesse sentido, a Organização Mundial da Saúde (OMS) estabelece recomendações de boas práticas na atenção ao parto, que visam limitar a quantidade dessas intervenções. Quando se trata da cesárea, para a OMS, não há evidências que justifiquem taxas maiores do que 10 a 15\%, já que esse aumento não se associa à redução da mortalidade materna (World Health Organization [WHO], 2015). Quanto à episiotomia, ainda há divergência sobre a taxa ideal de uso, porém, acredita-se que números mais baixos do que $10 \%$ se associem a menor prejuízo para a gestante e ao recém-nascido (WHO, 1996).

Ao se analisar o quadro geral dos nascimentos no Brasil, em 2018 a cesárea representou 57,9\% de todos os partos. Seguindo essa tendência, o Estado de Santa Catarina teve, no mesmo, ano a taxa de 57,2\% de cesáreas (MS, 2018). Já a episiotomia foi utilizada em 61,8\% dos partos na cidade de Chapecó no ano de 2016 (Schmalfuss, Rossetto, Baseggio, Radichewski, \& Batista, 2019). A maior ocorrência de cesárea e de intervenções desnecessárias poderiam estar relacionadas tanto a características obstétricas da gestante quanto a questões sociodemográficas, culturais e de assistência que influenciam essas escolhas (Schmalfuss et al., 2019; Rasador \& Abegg, 2019).

Considerando-se esse contexto, mostra-se importante conhecer de modo mais aprofundado o panorama dos partos e nascimentos e, consequentemente, compreender quais fatores que os influenciam e os definem. Dessa maneira, esta pesquisa 
tem como objetivo descrever as características sociodemográficas e clínicas de acordo com o tipo de parto (vaginal ou cesárea) e o tipo de convênio (SUS ou privado) de uma coorte de puérperas em um hospital público da região oeste de SC.

\section{Metodologia}

Trata-se de um estudo descritivo, transversal e retrospectivo, realizado a partir da base de dados de uma coorte de puérperas com partos vaginais ou cesáreas atendidas em um hospital da região oeste de Santa Catarina, referência para o atendimento na região. A coorte tem como objetivo de estudo avaliar a incidência de infecção puerperal a partir da exposição ao parto vaginal ou cesárea.

A coleta dos dados ocorreu entre o período de novembro de 2019 e março de 2020 e foi feita a partir do registro dos partos vaginais e cesáreas realizados na instituição entre os meses de maio e julho de 2019. Para a coleta de dados, foram utilizados os registros dos prontuários das pacientes, que se encontravam digitalizados e armazenados na rede interna do hospital. Os prontuários foram acessados no setor de arquivo por dois estudantes, em horários pré-agendados, e copiados para planilhas digitais, para posterior análise.

Foram excluídas do estudo mulheres menores de 18 anos, residentes em outros municípios e parturientes de natimortos, além de terem sido desconsideradas as 10 primeiras parturientes da lista, como parte do teste piloto no início da coleta dos dados.

O tamanho amostral, calculado para o estudo de base, foi realizado através do programa EpiInfo (software gratuito e de domínio público criado pelo CDC - Centers for Disease Control and Prevention, em português Centro para o Controle e Prevenção de Doenças). Considerando o total de 300 partos por mês realizados no hospital pesquisado, por ano, estima-se que ocorram 3.600 partos. A partir desses dados e de percentuais de infecção puerperal encontrados na literatura $(2,92 \%)$ (Guimarães, Chianca, \& Oliveira, 2007), foi calculada uma amostra de 380 puérperas, sendo 190 que tiveram parto vaginal e 190 que realizaram cesárea, isso considerando um erro amostral de 5\% e um nível de confiança de 95\%.

As variáveis analisadas nos prontuários foram: raça/cor/etnia e idade materna, estado civil, tipo de convênio (SUS ou privado), número de gestações, via do parto, episiotomia, laceração (categorizada em primeiro ou segundo grau), comorbidades, intercorrência no parto e antibioticoprofilaxia. Quanto às comorbidades, foram consideradas quaisquer tipos de doença materna, como por exemplo infecção do trato urinário de repetição, toxoplasmose, hepatites e hipotireoidismo. Entretanto, para análise mais aprofundada foram consideradas apenas as doenças que apareceram com maior significância, que são as seguintes: diabetes mellitus, diabetes mellitus gestacional, hipertensão arterial prévia e doença hipertensiva específica da gestação e sífilis.

A análise foi realizada com o uso do software Statistical Package for the Social Sciences (SPSS), versão 18.0. Para as comparações entre os grupos, utilizou-se o teste do Qui-quadrado de Pearson ou o teste exato de Fisher, e o teste T-student para amostras independentes.

Este artigo deriva do projeto de pesquisa intitulado "Incidência de infecção puerperal em mulheres que realizaram parto vaginal com ou sem episiotomia na cidade de Chapecó".

Quanto aos cuidados éticos em pesquisa, o estudo teve aprovação pelo Comitê de Ética em Pesquisa da Universidade Federal da Fronteira Sul (CEP - UFFS) sob o protocolo CAAE n ${ }^{17032119.1 .0000 .5564 . ~}$ 


\section{Resultados}

Foram incluídas 460 mulheres na coorte, sendo 230 do grupo parto vaginal e 230 de cesárea. As características sociodemográficas e clínicas das parturientes estão descritas na Tabela 1.

Tabela 1 - Características sociodemográficas e clínicas das parturientes.

\begin{tabular}{|c|c|c|}
\hline & $\mathbf{n}$ & $(\%)$ \\
\hline \multicolumn{3}{|l|}{ Idade } \\
\hline até 20 anos & 60 & 13 \\
\hline 21 a 25 anos & 125 & 27,2 \\
\hline 26 a 30 anos & 131 & 28,5 \\
\hline 31 a 35 anos & 84 & 18,3 \\
\hline 36 a 40 anos & 50 & 10,9 \\
\hline 40 anos ou mais & 10 & 2,2 \\
\hline \multicolumn{3}{|l|}{ Raça/cor } \\
\hline Branca & 418 & 91,7 \\
\hline Preta & 17 & 3,7 \\
\hline Parda & 14 & 3,1 \\
\hline Indígena & 3 & 0,7 \\
\hline Amarela & 4 & 0,9 \\
\hline \multicolumn{3}{|l|}{ Estado Civil } \\
\hline Casada/União estável & 404 & 87,8 \\
\hline Divorciada & 4 & 0,9 \\
\hline Solteira & 51 & 11,1 \\
\hline Viúva & 1 & 0,2 \\
\hline \multicolumn{3}{|l|}{ Gestações } \\
\hline Primeira gestação & 145 & 31,8 \\
\hline Segunda gestação & 157 & 34,4 \\
\hline Terceira ou mais & 154 & 33,8 \\
\hline \multicolumn{3}{|l|}{ Aborto prévio } \\
\hline Um aborto ou mais & 69 & 15,1 \\
\hline Nenhum aborto & 387 & 84,9 \\
\hline \multicolumn{3}{|c|}{ Alguma comorbidade na gestação atual } \\
\hline Sim & 132 & 51,8 \\
\hline Não & 123 & 48,2 \\
\hline \multicolumn{3}{|c|}{ Intercorrência no parto } \\
\hline $\operatorname{Sim}$ & 11 & 2,6 \\
\hline Não & 414 & 97,4 \\
\hline \multicolumn{3}{|c|}{ Antibiótico na internação } \\
\hline Sim & 174 & 37,9 \\
\hline
\end{tabular}


Não

\section{Convênio}

SUS

Privado

Episiotomia

$\operatorname{Sim}$

Não

Laceração

Não

Sim, $1^{\circ} \mathrm{Grau}$

Sim, $2^{\circ} \mathrm{Grau}$
285

356

80

188

Total
81,7

18,3

24

11,3

88,7

62,1

46,4

29,9

23,7

50

100

Fonte: Elaborada pelos autores com base nos dados da pesquisa.

Quanto às características sociodemográficas, o grupo das mulheres entre 26 e 30 anos predominou (28,5\%), além disso, no que se refere ao número de gestações, 34,4\% estava na segunda e 33,8\% na terceira gestação. A maioria era de raça/cor branca $(91,7 \%)$ e estava casada ou em união estável $(87,8 \%)$. Referente às características clínicas, $15,1 \%$ teve abortamento prévio e 51,8\% alguma comorbidade na gestação. Durante o parto, 2,6\% das mulheres tiveram algum tipo de intercorrência e 37,9\% usaram antibiótico durante a internação. Quanto ao convênio utilizado, 81,7\% das mulheres usaram o SUS e, das mulheres que tiveram parto vaginal, em 11,3\% foi realizada a episiotomia, já nas que não foram submetidas a esse procedimento $29,9 \%$ tiveram laceração de primeiro grau e 23,7\% apresentaram laceração de segundo grau.

$\mathrm{Na}$ Tabela 2 estão descritas as características sociodemográficas e clínicas de acordo com o tipo de parto (vaginal ou cesárea). As variáveis que estiveram associadas ao tipo de parto com o p significativo <0,001 foram idade, alguma comorbidade na gestação atual, diabetes, hipertensão, uso de antibiótico na internação e tipo de convênio.

Tabela 2 - Comparação entre características sociodemográficas e clínicas pelo tipo de parto.

\begin{tabular}{|c|c|c|c|}
\hline & Vaginal n (\%) & Cesárea n (\%) & p-value \\
\hline Idade & & & $<0,001 *$ \\
\hline até 20 anos & $45(75)$ & $15(25)$ & \\
\hline 21 a 25 anos & $73(58,4)$ & $52(41,6)$ & \\
\hline 26 a 30 anos & $57(43,5)$ & $74(56,5)$ & \\
\hline 31 a 35 anos & $38(45,2)$ & $46(54,8)$ & \\
\hline 36 a 40 anos & $15(30)$ & $35(70)$ & \\
\hline 40 anos ou mais & $2(20)$ & $8(80)$ & \\
\hline Raça/cor & & & $0,436^{*}$ \\
\hline Branca & $206(49,3)$ & $212(50,7)$ & \\
\hline Negra & $14(54,8)$ & $14(45,2)$ & \\
\hline Amarela ou indígena & $5(71,4)$ & $2(28,6)$ & \\
\hline Estado Civil & & & $0,669 * *$ \\
\hline
\end{tabular}


Casada/União estável

Divorciada/Solteira/Viúva

\section{Gestações}

Primeira gestação

Segunda gestação

Terceira ou mais

\section{Aborto prévio}

Um aborto ou mais

Nenhum aborto

\section{Alguma comorbidade na gestação atual}

Sim

Não

Sífilis

Sim

Não

Diabetes

Diabete Mellitus

Diabete Mellitus Gestacional

Não

\section{Hipertensão}

HAS prévia

Doença hipertensiva da gestação

Não

\section{Antibiótico na internação}

Sim

Não

\section{Intercorrência no parto}

Sim

Não

Convênio

SUS

Privado

Total

$$
200(49,5)
$$$$
30(53,6)
$$

$77(49)$

$78(50,6)$

$37(53,6)$

192(49,6)

$51(38,6)$

90(73,2)

$13(81,2)$

$128(53,6)$

2(33,3)

$3(14,3)$

$136(59,6)$

2(16,7)

$0(0)$

$139(59,4)$

21(12,1)

208(73)

$8(72,7)$

$221(53,4)$

202(56,7)

4(5)

230
204(50,5)

26(46,4)

$0,934 *$

71(49)

$80(51)$

$76(49,4)$

$0,602 * *$

$32(46,4)$

$195(50,4)$

$<0,001 * *$

$81(61,4)$

$33(26,8)$

$0,038 * *$

$3(18,8)$

$111(46,4)$

$<0,001^{*}$

$4(66,7)$

$18(85,7)$

92(40,4)

$<0,001^{*}$

10(83,3)

9(100)

$95(40,6)$

$<0,001 * *$

153(87,9)

$77(27)$

$0,236 * *$

$3(27,3)$

193(46,6)

$<0,001 * *$

154(43,3)

76(95)

230

* Qui-quadrado de Pearson

** Qui-quadrado pelo Teste Exato de Fischer

Totais podem variar devido à possibilidade de não resposta

Fonte: Elaborada pelos autores com base nos dados da pesquisa

Na Tabela 3 estão descritas as características sociodemográficas e clínicas de acordo com o tipo de convênio (SUS ou privado). As variáveis que estiveram associadas ao tipo de convênio com o p significativo $<0,005$ foram idade, número de gestações, uso de antibiótico na internação, tipo de parto e alguma comorbidade na gestação atual. 
Tabela 3 - Comparação entre características sociodemográficas e clínicas pelo tipo de convênio.

\begin{tabular}{|c|c|c|c|}
\hline & SUS n (\%) & Privado n (\%) & p-value \\
\hline Idade & & & $<0,001 *$ \\
\hline até 20 anos & $49(13,8)$ & $5(6,3)$ & \\
\hline 21 a 25 anos & $109(30,6)$ & $9(11,3)$ & \\
\hline 26 a 30 anos & $99(27,8)$ & $27(33,8)$ & \\
\hline 31 a 35 anos & $61(17,1)$ & $19(23,8)$ & \\
\hline 36 a 40 anos & $30(8,4)$ & $18(22,5)$ & \\
\hline 40 anos ou mais & $8(2,2)$ & $2(2,5)$ & \\
\hline Raça/cor & & & $0,220 *$ \\
\hline Branca & $319(90,4)$ & $76(96,2)$ & \\
\hline Negra & $28(7,9)$ & $2(2,5)$ & \\
\hline Amarela ou indígena & $6(1,7)$ & $1(1,3)$ & \\
\hline Estado Civil & & & $0,251 *$ \\
\hline Casada/União estável & $310(87,1)$ & $74(92,5)$ & \\
\hline Divorciada/Solteira/Viúva & $46(12,9)$ & $6(7,5)$ & \\
\hline Gestações & & & $<0,001 *$ \\
\hline Primeira gestação & $106(29,9)$ & $31(40,3)$ & \\
\hline Segunda gestação & $112(31,5)$ & $35(45,5)$ & \\
\hline Terceira ou mais & $137(38,6)$ & $11(14,3)$ & \\
\hline Aborto prévio & & & $0,482 * *$ \\
\hline Um aborto ou mais & $56(15,8)$ & $9(11,7)$ & \\
\hline Nenhum aborto & $299(84,2)$ & $68(88,3)$ & \\
\hline Alguma comorbidade na gestação atual & & & $0,002 * *$ \\
\hline Sim & $107(49,1)$ & $17(85)$ & \\
\hline Não & $111(50,9)$ & $3(15)$ & \\
\hline Sífilis & & & $0,622 * *$ \\
\hline $\operatorname{Sim}$ & $15(6,9)$ & $0(0)$ & \\
\hline Não & $203(93,1)$ & $20(100)$ & \\
\hline Diabetes & & & $0,364 *$ \\
\hline $\mathrm{DM}$ & $4(1,8)$ & $1(5)$ & \\
\hline $\mathrm{DMG}$ & $18(8,3)$ & $3(15)$ & \\
\hline Não & $196(89,9)$ & $16(80)$ & \\
\hline Hipertensão & & & $0,377 *$ \\
\hline HAS prévia & $8(3,7)$ & $2(10)$ & \\
\hline Doença hipertensiva da gestação & $8(3,7)$ & $1(5)$ & \\
\hline Não & $202(92,7)$ & $17(85)$ & \\
\hline Tipo de parto & & & $<0,001 * *$ \\
\hline Vaginal & $202(56,7)$ & $4(5)$ & \\
\hline Cesárea & $154(43,3)$ & $76(95)$ & \\
\hline
\end{tabular}


Episiotomia

Sim

Não

Laceração

Não

Sim, $1^{\circ} \mathrm{Grau}$

Sim, $2^{\circ} \mathrm{Grau}$

Antibiótico na internação

Sim

Não

Intercorrência no parto

Sim

Não

Total

20(10,9)

164(89,1)

$86(46,7)$

$54(29,3)$

44(23,9)

126(35,5)

$229(64,5)$

$9(2,7)$

$325(97,3)$
$0,068 * *$

2(50)

2(50)

$0,612 *$

2(66,7)

$1(33,3)$

$0(0)$

$<0,001 * *$

$47(58,8)$

$33(41,3)$

$1(1,5)$

$66(98,5)$

* Qui-quadrado de Pearson

** Qui-quadrado pelo Teste Exato de Fischer

Totais podem variar devido possibilidade de não resposta

Fonte: Elaborada pelos autores com base nos dados da pesquisa

\section{Discussão}

Diante dos resultados apresentados, é possível delinear um panorama dos partos em um hospital público da região oeste de Santa Catarina. Quanto ao perfil, predominou o grupo das mulheres que foi atendida pelo SUS, tinha raça/cor branca, estava com idade entre 21 a 30 anos, era casada e estava em sua segunda ou terceira gestação. Tais achados foram semelhantes aos de outros estudos brasileiros que também apontam predomínio de parturientes nessa faixa etária e com esse perfil, sendo demonstrada uma correlação com melhor desenvolvimento da gestação nesses casos (Silva \& Pelloso, 2009; Andrade et al., 2018; Freitas \& Fernandes, 2016).

Observa-se aumento do número de cesáreas quanto maior a idade materna entre usuárias do sistema público de saúde, além da proporção ser maior em mulheres brancas (Rattner \& Moura, 2016). Mais precisamente, infere-se que o parto vaginal foi a via mais comumente utilizada pelas mulheres até os 25 anos. Após essa faixa etária, a cesárea torna-se a via de escolha para a realização do parto. Tal cenário também foi encontrado em outras pesquisas sobre o tema que, além disso, indicam haver ainda uma correlação do aumento do número de cesáreas quanto maior a escolaridade das pacientes (Rattner \& Moura, 2016; Barros et al., 2011; Sales, Quitete, Knupp, \& Martins, 2020). Com relação ao estado civil, a maior prevalência de mulheres casadas ou em união estável seguiu a tendência de outros estudos, apesar de apresentar porcentagem um pouco mais elevada na pesquisa desenvolvida (Rasador \& Abegg, 2019; Freitas \& Fernandes, 2016; Barbosa et al., 2017). Nesse sentido, a situação conjugal estável é apontada como um fator relevante, especialmente porque pode proporcionar maior segurança e aumento do apoio para a puérpera (MS, 2012).

$\mathrm{O}$ atendimento privado esteve relacionado a uma maior idade e ao menor número de gestações prévias, sendo esses dados convergentes aos de outras pesquisas (Freitas \& Fernandes, 2016). Segundo a literatura, usuárias do sistema privado de saúde possuem maior escolaridade e capacidade econômica em comparação às atendidas pelo SUS (Freitas \& Fernandes, 2016; Fontenelle, 2017). Tais variáveis possuem relação com diversas características socioeconômicas, dentre elas o rendimento, tempo de escolaridade e ocupação, fatores inversamente proporcionais à fecundidade (Filho \& Reichert, 2017). 
Os dados que representam a utilização da episiotomia nos partos expõem que essa técnica foi empregada em 11,3\% dos casos, taxa consideravelmente menor do que aquela registrada por um estudo realizado na mesma instituição hospitalar no ano de 2016, quando foi encontrado que 61,8\% das parturientes foram submetidas a esse procedimento (Schmalfuss et al., 2019). Percebe-se, então, uma drástica redução no emprego dessa conduta, o que representa um avanço na assistência ao parto, tendo em vista que estudos apontam que seu uso indiscriminado não demonstra ser benéfico à gestante e ao recém-nascido, além de poder gerar complicações no parto e consequências irreversíveis, como dispareunia e incontinência fecal e urinária (Jiang et al., 2017).

Outro fator importante desse dado é a sua a proximidade ao índice sugerido pela OMS, organismo que, apesar de não definir metas, indica que índices próximos de $10 \%$ de uso da episiotomia estão relacionados a menores prejuízos maternos e ao recém-nascido (WHO, 2015). Dessa maneira, é possível inferir que, apesar de sempre haver espaço para aprimoramentos na assistência ao parto, a diminuição da taxa de episiotomia e sua proximidade com os parâmetros recomendados denotam evolução nesse sentido.

Em complemento, neste estudo, mesmo com taxas mais baixas de episiotomia, foi encontrado que $46,4 \%$ das mulheres submetidas ao parto não sofreram qualquer laceração. Entre as 53,6\% que sofreram a referida lesão, 26,9\% foi de primeiro grau e $23,7 \%$ de segundo grau. Esse valor está abaixo do que é encontrado na literatura, que demonstra que, aproximadamente, $85 \%$ das mulheres que realizam parto vaginal terão algum grau de laceração, observando um decréscimo dessa porcentagem nas parturientes multíparas, quando as lacerações ocorrem em cerca de 68,8\% das pacientes (Australian Journal of General Practice [AJGP], 2018).

É possível supor uma relação entre essas taxas mais baixas de laceração e de lesões menos graves com a realização da episiotomia na instituição. Isso porque a literatura demonstra que, quando bem aplicada e obedecendo aos critérios de realização, a episiotomia promove proteção ao períneo, evitando lacerações mais graves (AJGP, 2018; Royal College of Obstetricians and Gynaecologists [RCOG], 2015). No entanto, são necessários estudos mais aprofundados sobre o tema na instituição para que seja possível afirmar essa associação.

No que se refere às condições clínicas, as pacientes que possuíam alguma comorbidade no momento da internação, 61,4\% foram submetidas à cesárea. Esse número é ainda maior ao analisar especificamente os casos em que as pacientes tinham diabetes mellitus gestacional, hipertensão arterial ou doença hipertensiva específica da gestação, em que a cesárea ocorreu em 66,7\%; 83,3\% e 100\% dos casos, respectivamente. Outros estudos também mostram maior associação da cesárea em gestações de risco, no entanto, as recomendações atuais não instituem essa via como uma indicação absoluta para esses casos, devendo a decisão acerca da via de parto ser tomada a partir da análise de cada situação e considerando outros fatores, como idade gestacional e urgência (Rasador \& Abegg, 2019; Oliveira, Melo, Novaes, Ferracioli, \& Mathias, 2016; WHO, 2018).

Em relação à prevalência de comorbidades, notou-se que $85 \%$ das pacientes atendidas por financiamento privado tinham alguma condição clínica no momento do parto. Esse número está em consonância com o que foi encontrado em um trabalho que analisou as intercorrências na gravidez em puérperas brasileiras, cuja taxa verificada foi de 87,8\% (Varela, Oliveira, Melo, \& Mathias, 2017). A elevada taxa de comorbidades percebida neste estudo entre as mulheres atendidas pela rede privada pode ser parcialmente explicada pelo fato dessas parturientes possuírem uma idade mais avançada; praticamente a metade delas tinha entre 31 e 40 anos, o que propicia uma maior prevalência de doenças crônicas não transmissíveis e um maior risco gestacional (MS, 2012).

Quanto à antibioticoterapia, o medicamento foi utilizado em 87,9\% das internações que resultaram em cesárea. Esse número se deve ao fato de a cesárea representar o principal fator de risco para o desenvolvimento de infecção puerperal, portanto, a profilaxia com antibióticos deve ser instituída para todas as pacientes nesse tipo de parto, a não ser que a parturiente 
já esteja fazendo uso dessas drogas devido a outra patologia (Agência Nacional de Vigilância Sanitária [ANVISA], 2017; Federação Brasileira das Associações de Ginecologia e Obstetrícia [FEBRASGO], 2016). Evidenciou-se também o maior uso de antibioticoterapia nos atendimentos privados, entretanto é preciso ressaltar que 95\% das mulheres realizaram cesárea, e esse procedimento por si só está associado ao aumento do uso de antibióticos e outros tipos de fármacos durante a internação (Silveira et al., 2019). Sobre o uso no parto vaginal, como não há recomendação para profilaxia antibiótica em partos sem complicações, infere-se que essa taxa de uso seja explicada, por exemplo, em casos de administração intraparto para mulheres portadoras de Estreptococos do grupo B ou sífilis (Procianoy \& Silveira, 2020; MS, 2019).

No que tange à relação entre a via de parto e o tipo de convênio, parturientes que utilizaram o Sistema Único de Saúde, em sua maioria, tiveram parto vaginal (56,7\%), enquanto que 43,3\% foram submetidas à cesárea. Apesar da taxa de cesárea estar um pouco abaixo da tendência para o Estado de Santa Catarina, que teve, em 2018, 57,2\% dos partos por essa via, ela confirma os altos índices e o afastamento da taxa de 15\% recomendada pela OMS (WHO, 2015; MS, 2018). Esse distanciamento se torna ainda mais evidente quando se analisam as pacientes da rede privada na instituição, já que os achados demonstram que $95 \%$ dessas realizaram cesárea. Tal quadro se mostra frequente na literatura, que aponta taxas que variam de 83,9\% na região nordeste do Rio Grande do Sul, 89\% em Florianópolis/SC e 93,8\% em Maringá/PR (Rasador \& Abegg, 2019; Freitas \& Fernandes, 2016; Oliveira et al., 2016). Esses altos índices estão em consonância com o nacional, que, em 2018, apontou a cesárea como 57,9\% dos partos, figurando, assim, como o segundo país que mais realizou essa cirurgia no mundo naquele ano (MS, 2018; FEBRASGO, 2018).

Não foi possível definir o motivo da alta taxa de cesárea no sistema privado nesta pesquisa, no entanto, um estudo anterior que também encontrou esse cenário aponta que isso se deve ao fato da parturiente ter maior poder de decisão nesse modelo de assistência. Além disso, a preferência pela cesárea eletiva foi relacionada a diversos aspectos culturais e a comodidades que essa escolha proporciona, como a possibilidade de planejamento da data, além de crenças quanto ao parto vaginal, como o medo da dor do parto associado ao mito de que não é feita analgesia e o receio de alterações anatômicas vaginais no pós-parto (Oliveira et al., 2016).

Ainda quanto à correlação entre tipo de parto e convênio, 43,3\% dos partos realizados pelo SUS foram cesáreas, índice semelhante aos encontrados por outros estudos (Freitas \& Fernandes, 2016; Oliveira et al., 2016). Portanto, ainda que esse número seja consideravelmente menor do que o da rede privada, cabe assinalar que a realização de cesárea pelo serviço público também se encontra acima dos índices recomendados pela OMS.

Por fim, tem-se como limitações do estudo a não inclusão de mulheres menores de 18 anos de idade na análise, assim como a ausência de informações sobre renda e escolaridade, que não constavam em prontuário. Ademais, não foram realizados cálculos de proporcionalidade entre as raças/etnias abordadas no estudo, o que impede comparações entre essas categorias.

\section{Conclusão}

A partir da amostra estudada e dos dados relativos aos partos no local e no período pesquisado, é possível traçar um delineamento geral desse cenário, identificando-se que a maioria das mulheres se encontrava na faixa etária entre 21 e 30 anos, pertencia à raça branca, era casada ou estava em união estável, gestava pela segunda ou terceira vez e foi atendida pelo Sistema Único de Saúde.

Observou-se que quase a totalidade das mulheres atendidas pela rede privada foram submetidas a cesárea e, entre as que receberam esse atendimento, a maioria era branca. Mais precisamente, infere-se enorme disparidade na comparação do procedimento utilizado na rede pública e mediante atendimento privado, evidenciando ínfimo número de partos vaginais neste último. Nesse ponto, sublinha-se a constatação do uso excessivo de cesárea, acarretando em uma taxa superior àquela 
recomendada pela Organização Mundial da Saúde, principalmente nos atendimentos do setor privado. Nesse ponto, é importante lembrar que a cesárea desnecessária é considerada uma problemática no país e que, embora esse procedimento tenha um baixo risco de complicações, ao ser realizado de forma infrene, o resultado é um número considerável de intercorrências cirúrgicas graves.

Diferentemente dos índices de cesáreas, notou-se que o índice de episiotomia excedeu discretamente a taxa recomendada pela OMS, que é de cerca de 10\%. Esse procedimento, que ainda não possui evidências científicas suficientes para estabelecer quais seriam as suas reais indicações, foi durante muito tempo empregado de forma indiscriminada e rotineira acarretando prejuízos às mulheres a ele submetidas.

Acredita-se que os resultados deste estudo e de outros que aprofundem a temática possam contribuir para a melhor compreensão das características dos partos na região oeste de Santa Catarina e do Brasil, bem como propiciar maior conhecimento acerca do perfil das pacientes que passam por esse momento e, desse modo, auxiliar para a melhoria da assistência ao parto, mediante a orientação na formulação e execução de práticas profissionais e de políticas públicas voltadas à essas mulheres.

\section{Referências}

Agência Nacional de Vigilância Sanitária (2017). Medidas de Prevenção e Critérios Diagnósticos de Infecções Puerperais em Parto Vaginal e Cirurgia Cesariana. Agência Nacional de Vigilância Sanitária: Anvisa.

Andrade, S. G. de., Vasconcelos, Y. A. de., Carneiro, A. R. S., Severiano, A. R. G., Terceiro, A. J. de M. D., Silva, T. B. da., Carneiro, J. K. R., \& Oliveira, M. A. S. (2018). Perfil sociodemográfico, epidemiológico e obstétrico de parturientes em um hospital e maternidade de Sobral, Ceará. Revista Prevenção de Infecção e Saúde. 4. 10.26694/repis.v4i0.7283

Australian Journal of General Practice (2018). Perineal tears - A review. https://www1.racgp.org.au/ajgp/2018/january-february/perineal-tears-a-review

Barbosa, E. M., Oliveira, A. S. S. de, Galiza, D. D. F. de, Barros, V. L. de., Aguiar, V. F. de., \& Marques, M. B. (2017). Perfil Sociodemográfico e Obstétrico de Parturientes de um Hospital Público. Rev Rene, 18(2), 227-32. 10.15253/2175-6783.2017000200012

Barros, A. J. D., Santos, I. S., Matijasevich, A., Domingues, M. R., Silveira, M., Barros, F. C., \& Victora, C. G. (2011). Padrões de partos em uma coorte de nascimentos: cesarianas universais para os ricos. Revista de Saúde Pública. 45(4), 635-643. 10.1590/S0034-89102011005000039

Carneiro, L. M. de A., Paixão, G. P. do N., Sena, C. D., Souza, A. R. de., Silva, R. S. \& Pereira, A. (2015). Parto natural X parto cirúrgico: Percepções de mulheres que vivenciaram os dois momentos. Revista de Enfermagem do Centro-Oeste Mineiro. 5(2). 10.19175/recom.v0i0.744

Esteves-Pereira, A. P., Deneux-Tharaux, C., Nakamura-Pereira, M., Saucedo, M., Bouvier-Colle, M.-H., \& Leal, M. do C. (2016). Caesarean Delivery and Postpartum Maternal Mortality: A Population-Based Case Control Study in Brazil. PLoS ONE, 11(4). 10.1371/journal.pone.0153396

Federação Brasileira das Associações de Ginecologia e Obstetrícia (2016). Guia Prático: Infecções no Ciclo Grávido-Puerperal. https://www.febrasgo.org.br/media/k2/attachments/02-INFECCOyES_NO_CICLO_GRAVIDO_PUERPERAL.pdf

Federação Brasileira das Associações de Ginecologia e Obstetrícia (2018). Alta taxa de cesáreas no Brasil é tema de audiência pública. https://www.febrasgo.org.br/pt/noticias/item/728-alta-taxa-de-cesareas-no-brasil-e-tema-de-audiencia-

publica\#: :text=A\%C3\%A7\%C3\%A3o\%20do\%20MPF\%20pede\%20regulamenta\%C3\%A7\%C3\%A3o,consult \%C3\%B3rios\%20m\%C3\%A9dicos\%20e\%20h ospitais\%20privados.

Filho, P. J. M., \& Reichert, H. (2017). Condicionantes econômicas e sociais da fecundidade no Brasil. Ciências Sociais em Perspectiva. 16(30), 39-57. 10.5935/rcsp.v16i30.12179

Fontenelle, L. F (2017). Nível socioeconômico, cobertura por plano de saúde, e autoexclusão do Sistema Único de Saúde. Tese de doutorado. Programa de Pós-graduação em epidemiologia, Faculdade de Medicina, Universidade Federal de Pelotas, Pelotas, Brasil.

Freitas, P. F., \& Fernandes, T. M. B. (2016). Associação entre fatores institucionais, perfil da assistência ao parto e as taxas de cesariana em Santa Catarina. Revista Brasileira de Epidemiologia. 19(3), 525-538. 10.1590/1980-5497201600030005

Guimarães, E. E. R., Chianca, T. C. M. \& Oliveira, A. C. de. Infecção puerperal sob a ótica da assistência humanizada ao parto em maternidade pública. Revista Latino-Americana de Enfermagem. 15(4), 536-542. 10.1590/S0104-11692007000400003.

Jiang, H., Qian, X., Carroli, G., \& Garner, P. (2017). Selective versus routine use of episiotomy for vaginal birth. Cochrane Database of Systematic Reviews. 2. 10.1002/14651858.CD000081.pub3

Leal, M. do C., Bittencourt, S. de A., Esteves-Pereira, A. P., Ayres, B. V. da S., Silva, L. B. R. A. de A., Thomaz, E. B. A. F. \& Lamy, Z. C., et al. (2019). Avanços na assistência ao parto no Brasil: Resultados preliminares de dois estudos avaliativos. Cadernos de Saúde Pública, 35(7). 10.1590/0102311x00223018 
Ministério da Saúde (2012). Atenção ao pré-natal de baixo risco (Cadernos de Atenção Básica). Secretaria de Atenção à saúde. Departamento de Atenção Básica: Ministério da Saúde.

Ministério da Saúde. (2018). Estatísticas Vitais. Departamento de Informática do SUS: Ministério da Saúde.

Ministério da Saúde. (2017). Diretriz Nacional de Assistência ao Parto Normal. Secretaria de Ciência, Tecnologia e Insumos estratégicos. Comissão Nacional de Incorporação de Tecnologias no SUS: Ministério da Saúde.

Ministério da Saúde (2012). Gestação de alto risco: manual técnico (Série A. Normas e Manuais Técnicos). Secretaria de Atenção à Saúde. Departamento de Ações Programáticas Estratégicas: Ministério da Saúde.

Ministério da Saúde (2019). Protocolo Clínico e Diretrizes Terapêuticas para Prevenção da Transmissão Vertical do HIV, Sífilis e Hepatites Virais. Secretaria de Vigilância em Saúde. Departamento de Doenças de Conduções Crônicas e Infecções Sexualmente Transmissíveis: Ministério da Saúde.

Oliveira, R. R. de, Melo, E. C., Novaes, E. S., Ferracioli, P. L. R. V., \& Mathias, T. A. F. (2016). Fatores associados ao parto cesárea nos sistemas público e privado de atenção à saúde. Revista da Escola de Enfermagem da USP, 50(5), 733-740. http://dx.doi.org/10.1590/S0080-623420160000600004

Pascoal, J. M. F., \& Reichert H. (2017). Condicionantes econômicos e sociais da fecundidade no Brasil. Revista Ciências Sociais em Perspectiva, 16(30), 3957. https://doi.org/10.5935/rcsp.v16i30.12179

Procianoy, R. S., \& Silveira, R. C. (2019). Os desafios no manejo da sepse neonatal. Jornal de Pediatria, 96(S1), 80-86. https://doi.org/10.1016/j.jped.2019.10.004.

Rasador, S., \& Abegg C. (2019). Fatores associados à via de parto em um município da região nordeste do Estado do Rio Grande do Sul, Brasil. Revista Brasileira de Saúde Materno Infantil. 19(4), 797-815. 10.1590/1806-93042019000400004.

Rattner, D., \& Moura, E. C. de. (2016). Nascimentos no Brasil: Associação do tipo de parto com variáveis temporais e sociodemográficas. Revista Brasileira de Saúde Materno Infantil. 16(1), 39-47. 10.1590/1806-93042016000100005

Royal College of Obstetricians and Gynaecologists (2015). The Management of Third- and Fourth-Degree Perineal Tears. https://www.rcog.org.uk/globalassets/documents/guidelines/gtg-29.pdf

Sales, J. de L., Quitete, J. B., Knupp, V. M. de A. O., \& Martins, M. A. R. (2020). Assistência ao parto em um hospital da baixada litorânea do Rio de Janeiro: desafios para um parto respeitoso. Revista Online de Pesquisa (Universidade Federal do Estado do Rio de Janeiro). 12, 107-113. 10.9789/21755361.rpcfo.v12.7092

Schmalfuss, J. M., Rossetto, M., Baseggio, L., Radichewski, V., \& Batista, J. d'Arc L. (2016). Fatores associados ao relato de episiotomia em parturientes atendidas em um hospital do oeste catarinense. Revista de Enfermagem do Centro-Oeste Mineiro. 9(0). 10.19175/recom.v9i0.3294

Silva, G. F., \& Pelloso, S. M. (2009). Perfil das parturientes e seus recém-nascidos atendidos em um hospital-escola do Noroeste do Estado do Paraná. Revista da Escola de Enfermagem da USP. 43(1), 95-102. 10.1590/S0080-62342009000100012

Silveira, M. P. T., Miranda, V. I. A., Silveira, M. F. da., Pizzol, T. S. D., Mengue, S. S., \& Bertoldi, A. D. (2019). Uso de medicamentos na internação para o parto: coorte de nascimentos de Pelotas, 2015. Revista de Saúde Pública, 53(51). https://doi.org/10.11606/S1518-8787.2019053000913

Varela, P. L. R., Oliveira, R. R. de, Melo, E. C., \& Mathias, T. A. F. de. (2018). Intercorrências na gravidez em puérperas brasileiras atendidas nos sistemas público e privado de saúde. Revista Latino-Americana de Enfermagem, 25, e2949. https://doi.org/10.1590/1518-8345.2156.2949.

World Health Organization. (1996). Care in normal birth: a pratical guide. http://www.midwiferyservices.org/care_in_normal_birth_practical_guide.pdf

World Health Organization (2018). Policy of interventionist versus expectant management of severe pre-eclampsia before term. https://apps.who.int/iris/bitstream/handle/10665/277236/9789241550444-eng.pdf?ua=1

World Health Organization. (2015). WHO Statement on $\quad$ Caesarean Section https://www.who.int/reproductivehealth/publications/maternal_perinatal_health/cs-statement/en/ 